\title{
Case report: type 1 diabetes in monozygotic quadruplets
}

\author{
Katerina Stechova ${ }^{\star}, 1$, Zbynek Halbhuber ${ }^{2}$, Miluse Hubackova $^{1}$, Jana Kayserova ${ }^{3}$, Lenka Petruzelkova ${ }^{1}$, \\ Jana Vcelakova ${ }^{1}$, Stanislava Kolouskova ${ }^{1}$, Tereza Ulmannova ${ }^{1}$, Maria Faresjö ${ }^{4,5}$, Ales Neuwirth ${ }^{6}$, Radek Spisek $^{3}$, \\ Anna Sediva $^{3}$, Dominik Filipp ${ }^{6,7}$ and Zdenek Sumnik ${ }^{1,7}$
}

Type 1 diabetes (T1D) is an autoimmune disease characterized by the lack of insulin due to an autoimmune destruction of pancreatic beta cells. Here, we report a unique case of a family with naturally conceived quadruplets in which T1D was diagnosed in two quadruplets simultaneously. At the same time, the third quadruplet was diagnosed with the pre-diabetic stage. Remarkably, all four quadruplets were positive for anti-islet cell antibodies, GAD65 and IA-A2. Monozygotic status of the quadruplets was confirmed by testing 14 different short tandem repeat polymorphisms. Serological examination confirmed that all quadruplets and their father suffered from a recent enteroviral infection of EV68-71 serotype. To assess the nature of the molecular pathological processes contributing to the development of diabetes, immunocompetent cells isolated from all family members were characterized by gene expression arrays, immune-cell enumerations and cytokine-production assays. The microarray data provided evidence that viral infection, and IL-27 and IL-9 cytokine signalling contributed to the onset of T1D in two of the quadruplets. The propensity of stimulated immunocompetent cells from non-diabetic members of the family to secrete high level of IFN- $\alpha$ further corroborates this conclusion. The number of T regulatory cells as well as plasmacytoid and/or myeloid dendritic cells was found diminished in all family members. Thus, this unique family is a prime example for the support of the so-called 'fertile-field' hypothesis proposing that genetic predisposition to anti-islet autoimmunity is 'fertilized' and precipitated by a viral infection leading to a fully blown T1D.

European Journal of Human Genetics (2012) 20, 457-462; doi:10.1038/ejhg.2011.212; published online 23 November 2011

Keywords: type 1 diabetes; monozygotic quadruplets; enteroviral infection; gene expression array; cytokine array; genetic predisposition to diabetes

\section{INTRODUCTION}

Type 1 diabetes (T1D) is an autoimmune disease, the complexity of which is underlined by undesirable interactions between genes and environmental factors in genetically predisposed individuals. ${ }^{1}$ Although $\mathrm{T}$ cells are central to the mechanism of beta-cell destructions, ${ }^{2-5}$ a critical involvement of other cellular and humoral components of adaptive and innate immune system have also been demonstrated. ${ }^{1,6-8}$ Among environmental factors, infectious agents such as viruses primarily sensed by innate immune mechanisms are considered very potent triggers of T1D. ${ }^{9-12}$ Notably, and in this context, several recently published findings suggest a strong link between T1D and enteroviral infections. ${ }^{13-15}$

To prevent the clinical onset of T1D, immuno-intervention should be undertaken in the clinically silent pre-diabetic phase. However, it is difficult to identify suitable candidates for such interventions. ${ }^{16}$ The pre-diabetic phase is usually marked by the presence of autoantibodies to beta-cell antigens, ${ }^{17,18}$ but the presence of these antibodies alone is not sufficient to induce destruction of beta cells. $^{2}$
Here, we report a case of a family with monozygotic quadruplets where after an apparent enteroviral infection, two sisters were diagnosed with T1D while a third quadruplet was at pre-diabetic stage. To gain an insight into the molecular mechanism involved in the pathogenesis of this disease, all family members were studied for the presence of islet cell antibodies, gene expression profile of immune regulatory pathways, cellularity of $\mathrm{T}$ regulatory cells (Tregs) and dendritic cells (DCs), and cytokine responses.

\section{MATERIALS AND METHODS}

Family history

The quadruplets were born into a family with an older sibling after a physiological conception. Their development followed a typical path. At the age of 5 , two of these quadruplets were simultaneously diagnosed with T1D, while a third quadruplet was in a pre-diabetic phase. Interestingly, one month before the clinical manifestation, the two diabetic girls suffered from an apparent mild infection with respiratory symptoms. Laboratory tests showed that all quadruplets and their father suffered from an enteroviral infection. All family members were subjected to the glucose tolerance test one week after the diagnosis. Upon obtaining a parental informed consent, blood samples of all

\footnotetext{
${ }^{1}$ Department of Paediatrics, 2nd Medical Faculty of Charles University and University Hospital Motol, Prague, Czech Republic; ${ }^{2}$ Central European Biosystems, Prague, Czech Republic; ${ }^{3}$ Department of Immunology, 2nd Medical Faculty of Charles University and University Hospital Motol, Prague, Czech Republic; ${ }^{4}$ Department of Natural Sciences and Biomedicine, School of Health Sciences, Jönköping University, Jönköping, Sweden; ${ }^{5}$ Division of Paediatrics \& Diabetes Research Centre, Department of Clinical \& Experimental Medicine, Faculty of Health Sciences, Linkoping University, Linkoping, Sweden; ${ }^{6}$ Laboratory of Immunobiology, Institute of Molecular Genetics AS CR, Prague, Czech Republic ${ }^{*}$ Correspondence: Associated Professor K Stechova, Laboratory of Autoimmune Diseases, Department of Paediatrics, University Hospital Motol, V Uvalu 84, Prague 5 - Motol, 150 06, Czech Republic. Tel: +420 602194 803; Fax: +420 224432 020; E-mail: katerina.stechova@|fmotol.cuni.cz 7 These authors contributed equally to this work.
}

Received 14 June 2011; revised 13 October 2011; accepted 21 October 2011; published online 23 November 2011 
family members were collected and analysed. A family tree and clinical data are provided in Figure 1.

\section{HLA typing, confirmation of monozygosity and viral studies}

All family members were HLA typed using allele-specific primers. ${ }^{19}$ Serum samples from both the parents and the quadruplets were examined for signs of viral infections by ELISA, complement fixation and non-direct immunofluorescence. We focused on enteroviruses and coxsackie viruses as they are suspected to have a role in T1D development. ${ }^{13-15}$ Monozygotic status of the quadruplets was confirmed by testing 14 different short tandem repeat polymorphisms using Aneufast multiplex QF-PCR kit (molGENTIX, S.L., Barcelona, Spain), recommended by CODIS (Combined DNA Index System). ${ }^{20}$

\section{Immune cell isolation}

Peripheral-blood mononuclear cells (PBMCs) were isolated from heparinized venous blood by Ficoll-Paque density gradient centrifugation and cultured as described elsewhere. ${ }^{21}$ After $72 \mathrm{~h}$, cells were washed and subjected to RNA extraction and supernatants were frozen at $-80^{\circ} \mathrm{C}$ until used for cytokine analysis.

\section{Gene expression profiling}

RNA isolated from PBMCs was amplified and hybridized to a high-density gene array chip (NimbleGen, Roche-NimbleGen Inc., Madison, WI, USA) where 47633 human genome targets are presented with 8 probes per target. Chips were imaged (InnoScan 700 scanner, Innopsys, Carbonne, France), raw data were analysed using NimbleScan 2.4 software (Roche-NimbleGen Inc.) and used for further statistical analysis.

\section{Statistical and gene array analysis}

The goal of this analysis was to identify differences in the activity of the immune signalling pathways between the diabetic quadruplets and their nondiabetic siblings. The gene array statistical analysis was performed using the network linking (GeneSpring GX10, Agilent Technologies, Inc., Santa Clara, CA, USA) and the pathway analysis softwares (MetaCore, GeneGo, Inc., St Joseph, MO, USA). Using unpaired $t$-tests, only the differences in gene expression with $P$-values $<0.05$ were considered as statistically significant. The numbers used to generate the heat map represent normalized intensity values calculated as a $\log _{2}$ ratio of gene expression to the chip median expression value.
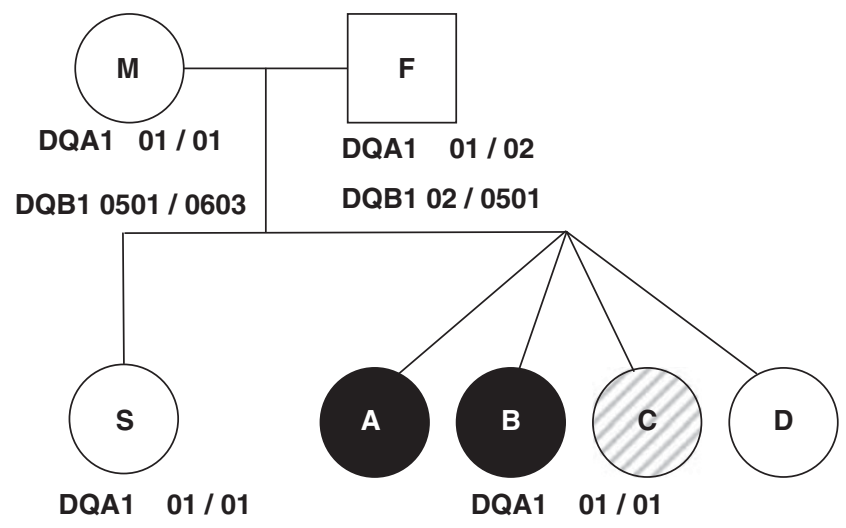

\section{DQB1 $0501 / 0603$}

DQB1 $0501 / 0501$

Figure 1 The family tree with indicated HLA DQ types. The quadruplets $(A-D)$ are genetically monozygotic and $D Q$ homozygous. The quadruplets $A$ and $B$ had simultaneous presentation of T1D just 1 month after an apparent enteroviral infection. The quadruplet $C$ is in the pre-diabetic phase. The quadruplet $D$ and an older sister (S) are diabetes free. All four quadruplets (A-D) produce anti-islet cell antibodies to GAD65 and IA-A2 autoantigens. HLA DQ types of mother $(M)$ and Father $(F)$ are also indicated.

\section{Cellularity of Tregs and DCs}

Tregs $\left(\mathrm{CD}^{+} \mathrm{CD}^{+} 5^{+} \mathrm{FoxP}^{+}\right)$and DCs (plasmacytoid, pDC as well as myeloid, $\mathrm{mDC}$ ) from freshly isolated PBMCs were counted and identified by flow cytometry (FACS) as described elsewhere. ${ }^{22}$ Family data were compared with the control samples derived from 23 healthy volunteers ( 12 females/11 males), median of age 18 years, range 14-26 years.

\section{PBMC cytokine responses}

To assess the cellular responses to Toll-like receptor 9 (TLR9) stimulation, $2 \times 10^{5}$ freshly isolated PBMCs were stimulated with ODN 2216 (Invivogen, Toulouse, France) for $24 \mathrm{~h}\left(37^{\circ} \mathrm{C}, 5 \% \mathrm{CO}_{2}\right)$ and cytokine productions were measured using multiplexed antibodies (Milliplex, Millipore, Billerica, MA, USA) on luminescent beads (Luminex 100 IS, Luminex Corporation, Austin, TX, USA). Similarly, a spontaneous secretion of cytokines was measured in the supernatants derived from PMBCs using a cytokine microarray (Ray Biotech, Norcross, GA, USA). Cytokine concentrations were compared with the relevant value ranges of the control population without statistical analysis.

\section{RESULTS}

Short tandem repeat polymorphisms test, HLA typing, anti-islet cell antibodies, glucose tolerance test and anti-viral antibodies All quadruplets exhibited identical results in all 14 short tandem repeat polymorphisms tested. The quadruplets are homozygous for HLA DQA 01 and DQB 0501 (Figure 1). The HLA class II alleles in the quadruplets are not associated with an increased risk for T1D in the Czech population. ${ }^{19}$ Remarkably, all four quadruplets were positive for anti-islet autoantibodies GAD65 and IA-2A, indicating an ongoing anti-islet autoimmunity in the non-diabetic quadruplets. The quadruplet $\mathrm{C}$ had a reduced first-phase insulin response and a 1-hour glucose concentration of $8.7 \mathrm{mmol} / \mathrm{l}$, a typical characteristic of a prediabetic stage. Only this quadruplet had an evidence of current Enterovirus 68-71 infection with IgM, IgA and IgG antibodies. The father and the other quadruplets had anamnestic Enterovirus 68-71 IgG antibodies only.

\section{Functional genomics}

Among the 47633 probe-sets tested, 2136 entities (with proved difference $P \leq 0.05$. and fold change $\geq 1$ ) were unique for the nondiabetic quadruplets and 2589 entities were typical of the two diabetic sisters. Out of 10 immune signalling networks with the largest differences in the gene expression profile between the diabetic and the non-diabetic quadruplets (Table 1), 4 are related to antiviral responses: (i) antiviral actions of interferons, (ii) TRIF-specific

Table 1 Ten most differently regulated immune signalling networks between the diabetic ( $A$ and $B$ ) and the non-diabetic ( $C$ and $D$ ) quadruplets

\begin{tabular}{lc}
\hline Signalling pathways & P-value \\
\hline Antiviral actions of interferons & 0.0003 \\
Role of TLR-3 and -4 in antiviral response: & 0.0011 \\
Trif-specific signalling pathways & \\
IFN alpha/beta signalling pathway & 0.0019 \\
IL-27 signalling pathway & 0.0034 \\
Antigen presentation by MHC class I & 0.0056 \\
IL-4-antiapoptotic action & 0.0141 \\
IL-12-induced IFN-gamma production & 0.0260 \\
IL-9 signalling pathway & 0.0359 \\
Human NKG2D signalling & 0.0366 \\
IL-4 signalling pathway & 0.0456
\end{tabular}


TLR3 and TLR4 signalling pathways, (iii) IFN alpha/beta signalling and (iv) antigen presentation by MHC class I. Important differences were also found in four other regulatory pathways related to the interleukin signalling and T-cell differentiation pathways (IL-4, -9, - 12 and -27). A gene cluster analysis of these pathways is presented in the Figure 2 where the gene expression for the diabetic and the nondiabetic quadruplets is compared.

\section{Cellularity of Tregs and DCs}

The relative number of Tregs was decreased in all family members in comparison with the value range of the control group (Supplementary Figure 3a). Similarly, the absolute numbers of pDCs and mDCs were also decreased compared with the mean of the control subjects (Supplementary Figure $3 \mathrm{~b}$ and $3 \mathrm{c}$, respectively). The only exception for the decrease in pDCs was the older sister and that for the decrease in $\mathrm{mDCs}$ was the quadruplet $\mathrm{B}$.

\section{Spontaneous and induced cytokine production by PBMCs}

PMBCs from the diabetic quadruplets produced high levels of TGF-betal and low or undetectable levels of IL-10. A completely opposite pattern of production of these two cytokines was observed in the pre-diabetic quadruplet C (Supplementary Figure 4). Remaining healthy members of the family produced intermediate to low levels of IL-10 and low to undetectable levels of TGF-beta1. All family members produced comparable levels of IL-13 (Supplementary Figure 4). Thus, IL-10/TGF-beta-1 imbalance between the diabetic and the non-diabetic members of the family was the most striking result of this analysis. In addition, we detected higher spontaneous levels of IFN- $\gamma$ and other Th1 cytokines mainly in the non-diabetic quadruplet D. Moreover, only the father's PBMCs produced detectable amounts of IL-17 (data not shown).

The stimulation of PBMCs with TLR9-specific ligand led to the increased production of IFN- $\alpha$ in the non-diabetic quadruplets, the older sister and the mother. Concentration of IFN- $\alpha$ was slightly increased in the diabetic siblings as opposed to the controls. In contrast, PBMCs from the father were refractory to TLR9 stimulation (Supplementary Figure 5).

\section{DISCUSSION}

A simultaneous clinical manifestation of T1D in siblings is rare. Taken into account that the probability of a natural conception of quadruplets is extremely low, ${ }^{23}$ the chance for the quadruplets to also be monozygotic and DQ homozygous is truly exceptional. Here, we documented a unique case of a family with naturally conceived quadruplets where two of them have already developed T1D and the third is in a pre-diabetic state with impaired first-phase insulin response in ivGTT. Moreover, all four quadruplets are positive for anti-GAD65 and IA-2A antibodies. Thus, the probability for an early onset of T1D in the two, so far non-diabetic quadruplets is quite high. $^{24}$

An interplay between genetic predisposition and environmental factors is implicated in T1D pathogenesis. ${ }^{25,26}$ External factors such as food, infections and stress factors should be taken into account. The quadruplets had the same duration of lactation and they have similar eating habits. They all attend the same kindergarten and follow a standard national vaccination programme. According to their medical records they did not suffer from any major childhood diseases and infections. The parents reported that both diabetic quadruplets showed visible signs of a mild respiratory infection just 1 month before the onset of diabetes. No other infection or medical conditions were reported before the onset of T1D. Serological examinations revealed that, indeed, all the quadruplets suffered from an enteroviral infection, still ongoing in the pre-diabetic quadruplet C. Viral infections are considered to have an important role in T1D pathogenesis, but the exact molecular mechanisms are still unknown. ${ }^{25,26}$ In this context, our data are consistent with the recently published metaanalysis of 33 prevalence studies suggesting that enterovirus infection is common among patients with T1D. ${ }^{27}$

Our microarray data provided additional evidence to support the notion that viral infection may have contributed to the onset of T1D in the two quadruplets. Notably, cellular anti-viral responses were more prominent in the diabetic sisters where the antiviral signalling of interferons was the most significantly affected pathway. In the diabetic and the non-diabetic sisters, three other virus-sensing pathways exhibited different activation status, namely, TRIF-dependent TLR3 and TLR4 antiviral responses, IFN $\alpha / \beta$ signalling and antigen presentation by MHCI. Various evolutionarily conserved microbial structures, so-called pathogen-associated molecular patterns, are recognized by pattern recognition receptors. Toll-like receptors (TLRs) represent a prototypical class of such receptors that recognize pathogen-associated molecular patterns. ${ }^{28}$ Moreover, TLR3 and TLR4 can signal the presence of viral RNAs and proteins, respectively. In addition, only TLR3 and 4 signal via adaptor protein TRIF, which triggers antiviral immune responses through the production of type I interferons $(\mathrm{IFN} \alpha / \beta)$ and inflammatory cytokines. ${ }^{29}$ TRIF signalling also leads to MyD88-independent DC maturation. ${ }^{30}$ In turn, interferons may not only significantly contribute to the development of Th1 immune responses considered as prodiabetogeneic ${ }^{31}$ but they can also cause the upregulation of MHC class I molecules on pancreatic beta cells, thus initiating the diabetogenic process itself. ${ }^{9,32}$ An alternative pathway able to transduce the detection of enteroviral infection to interferon $\alpha / \beta$ production involves the cytoplasmic helicase receptor MDA5 (melanoma differentiation-associated protein 5). ${ }^{33,34}$ A recent genome-wide association study implicated MDA5 in the pathogenesis of T1D. ${ }^{35}$ Thus, although we cannot preclude the contribution of other external factors and type of viruses, our serological and microarray data argue for a possible involvement of enteroviruses of EV68-71 serotypes in the development of T1D in our quadruplets. The propensity of stimulated PMBCs isolated from non-diabetic members of the family (with the exception of the father) to secrete high levels of IFN- $\alpha$ compared with the controls lends further support for this conclusion.

The other four pathways with the most prominent differences between the diabetic and the non-diabetic siblings are cytokine IL-27, -4, -12 and -9 signalling cascades affecting T-cell differentiation. Among these interleukins, the most promising candidates for diabetes-associated markers seem to be IL-27 and IL-9. Notably, it has been demonstrated that IL-27 functions as the key regulator of IL-10 and IL-17 production in human CD4 ${ }^{+} \mathrm{T}$ cells. ${ }^{36}$ Nowak and colleagues $^{37}$ also identified IL-9 as a mediator of Th17-driven inflammatory disease. Moreover, it has also been shown that IL-9 synergizes with TGF-beta1 to differentiate naive $\mathrm{CD}_{4}^{+} \mathrm{T}$ cells into Th17 cells and postulated an additional role for IL-9 in mediating the suppressive function of Tregs in experimental autoimmune encephalomyelitis. ${ }^{38}$ Thus, our microarray data are coherent with these findings and provide further support for the importance of IL-27 and IL-9 cytokine signalling in the development of T1D.

FACS analysis of freshly isolated PMBCs from all family members showed that the first-degree relatives of the diabetic quadruplets display a general decline in the number of pDCs and/or mDCs. Moreover, all family members displayed a lower number of naturally occurring Tregs. This is in agreement with a previously documented 


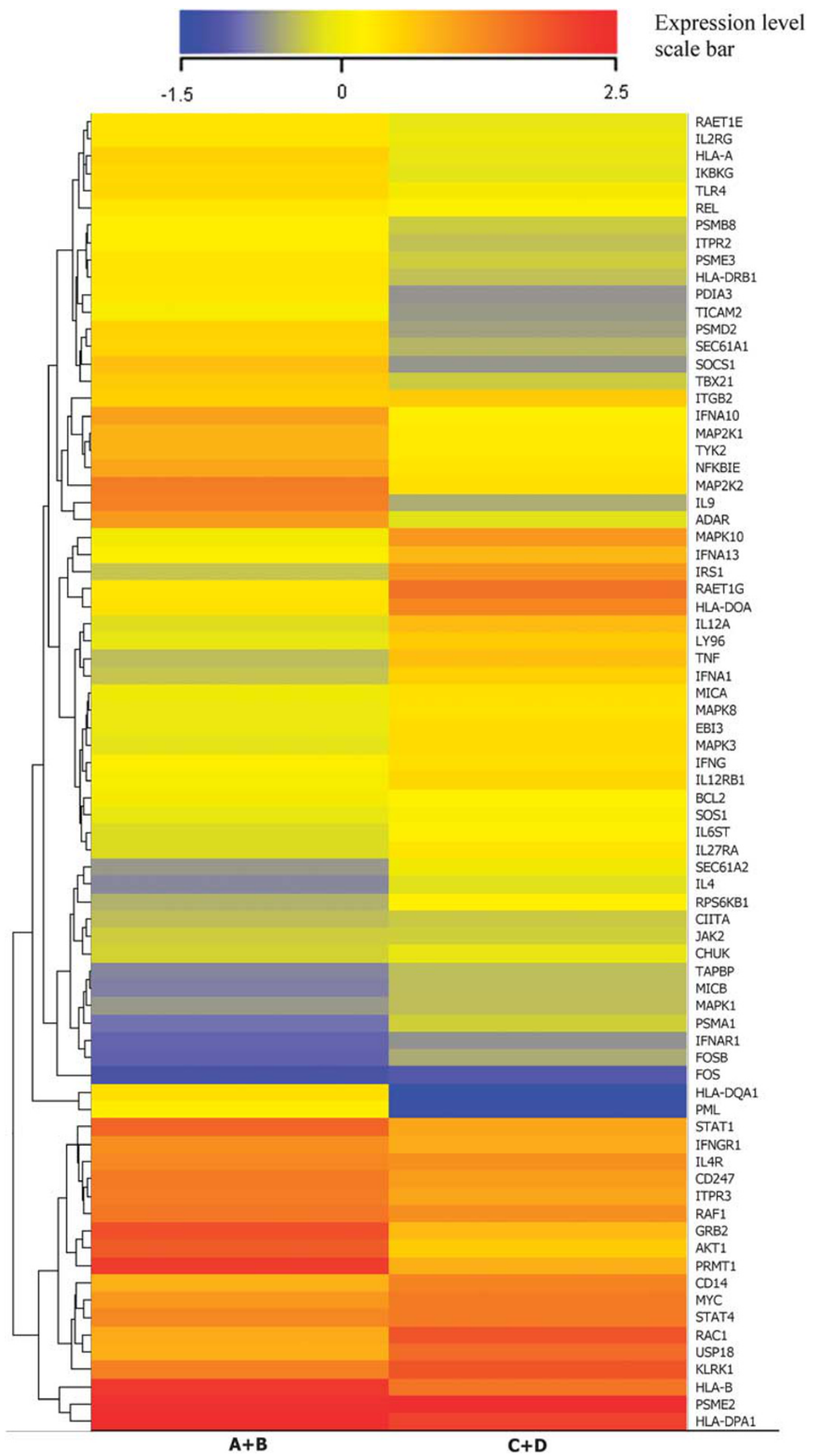

Figure 2 Microarray heat map of genes belonging to 10 most differentially regulated pathways between the diabetic and the non-diabetic quadruplets. A simplified view with averaged gene expression values of diabetic ( $A$ and $B$ ) and non-diabetic sisters $(C$ and $D)$ is presented. Red and blue indicate an increase and a decrease in the gene expression levels, respectively, as indicated in the scale bar. The affiliation of all listed genes with one of the ten signaling pathways listed in Table 1 is presented in the Supplementary Table. 
situation where siblings of T1D patients often reveal lower number of Tregs with impaired functional capabilities. ${ }^{39}$ We have also previously reported that PMBCs from young patients with T1D, predominantly produced Th3 cytokines IL-10 and TGF- $\beta \cdot{ }^{40,41}$ However, a predominant secretion of TGF- $\beta$ over IL-10 seen in our diabetic quadruplets has not been reported so far. Strikingly, this production pattern exhibited a reverse relation in their pre-diabetic sister. Although the mechanism underpinning this cytokine production pattern is not known at this time, it is suggestive of a switch in the regulation of cytokine expression during the transition from pre-diabetic to clinically manifested T1D phase.

The open question is then why, if all quadruplets show signs of enteroviral infection and concomitantly are positive for anti-islet autoantibodies GAD65 and IA-2A, only two of them have developed diabetes despite their identical genetic background? In this respect, the lack of information concerning the quadruplets' autoantibody positivity before enteroviral infection precluded us to establish direct causative links between the enteroviral seropositivity, islet autoimmunity and the onset of diabetes. ${ }^{42}$ Enteroviruses enter the body via ingestion and young children are their main target and reservoir. The incubation period lasts usually 3-6 days and symptoms of infection are usually subclinical, or very mild in the form of uncomplicated summer cold. Initially, enterovirus-specific antibodies of IgM class are detectable in 1-3 days after infection and are present for several months. After 7-10 days post-infection, a seroconversion to IgG class occurs and long lasting antiviral IgG antibodies, called anamnestic, are produced. ${ }^{43}$ The quadruplet $\mathrm{C}$ is the only individual from this family that is still serologically positive for the presence of enterovirusspecific IgM antibodies. Thus, although a pre-diabetic stage of the quadruplet $\mathrm{C}$ correlates with a more recent occurrence of enteroviral infection, its positivity for anti-islet autoantibodies GAD65 and IA-2A indicates that this infection might not be a primary cause of the antiislet autoimmunity, which rather results from a genetic predisposition of quadruplets to diabetes. ${ }^{44}$ This line of arguments is further supported by a recent study suggesting that the progression from islet autoimmunity to fully blown T1D may increase after an enterovirus infection. ${ }^{15}$ In this respect, the timing of infection and its periodic recurrence as well as a dose of the virus are certainly important parameters when considering the effect of viral infection on the same diabetes-susceptible genetic background. It is of note that a pair-wise concordance of T1D is $<40 \%$ among monozygotic twins (data from monozygotic quadruplets are not available) strongly advocating for a critical role of exogenous factors in the development of T1D. ${ }^{45,46}$ Taken altogether, the identical genetic background among quadruplets does not guarantee the synchronization of the onset of diabetes. In addition, an individual medical history and immunological experience reflected in differential level of gene hypermethylation could represent an important endogenous factor causing a discordance among genetically identical siblings. ${ }^{47}$ Thus, the correlation between our microarray data indicating an involvement of viral infection in the development of diabetes and the appearance of clinically apparent mild respiratory, possibly viral infection in two girls 1 month before clinical diagnosis of T1D is indicative that a viral infection contributed to the autodestructive diabetic process. Although the identity of this viral agent remains uncertain, EV68-71 serotypes positivity points to enteroviruses as obvious suspects. However, as we have no direct evidence linking enteroviruses to the onset of diabetes in these quadruplets, caution has to be exercised in the interpretation of these results.

In sum, this unique family case study provides a support for the socalled 'fertile-field' hypothesis proposing that genetic predisposition to anti-islet autoimmunity is 'fertilized' and precipitated by viral infections. $^{48}$ Our microarray data suggest that the viral activation of TRIF-mediated TLR signalling amplified by ensuing overproduction of IFN $\alpha / \beta$ cytokines could lead to an imbalance between anti- and pro-inflammatory signals towards the latter, resulting in a relatively rapid progression of T1D in genetically susceptible young quadruplets. ${ }^{18}$ Several lines of evidence also point to the contribution of enteroviral insult that preceded the clinically manifested onset of a fully blown and pre-diabetic stage of T1D in three out of four quadruplets. The finding that the remaining quadruplet is anti-islet autoantibody-positive, but still diabetes free with normal glucose tolerance test likely attests to her genetic predisposition to anti-islet autoimmunity. Thus, this family case is a prototypical example of the efficiency of environmental factors to cause disease in genetically identical predisposed individuals.

\section{CONFLICT OF INTEREST}

The authors declare no conflict of interest.

\section{ACKNOWLEDGEMENTS}

Gene expression study was exclusively supported by project NPVII 2B06019 from the Czech Ministry of Education. Studies on Tregs and DCs were supported by the project VZ MSM 0021620812 from the Ministry of Education, Youth and Sports of the Czech Republic, and by the project MZOFNM2005 from the Ministry of Health and by the project GACR P302/ $10 / 1679$. AN and DF as well as the cytokine analysis were supported by the project NPVII 2B08066 from the Czech Ministry of Education, Youth and Sports. This work was also partially supported by Grant AVOZ50520514 from the Academy of Sciences of the Czech Republic.

1 Todd JA: Etiology of type 1 diabetes. Immunity 2010; 32: 457-467.

2 Atkinson MA, Eisenbarth GS: Type 1 diabetes: new perspectives on disease pathogenesis and treatment. Lancet 2001; 358: 221-229.

3 Tree TI, Peakman M: Autoreactive T cells in human type 1 diabetes. Endocrinol Metab Clin North Am 2004; 33: 113-133.

4 Skowera A, Ellis RJ, Varela-Calviño $\mathrm{R}$ et al: CTLs are targeted to kill beta cells in patients with type 1 diabetes through recognition of a glucose-regulated preproinsulin epitope. J Clin Invest 2008; 118: 3390-3402.

5 Willcox A, Richardson SJ, Bone AJ et al: Analysis of islet inflammation in human type 1 diabetes. Clin Exp Immunol 2009; 155: 173-181.

6 Van Belle TL, Taylor P, von Herrath MG: Mouse models for type 1 diabetes. Drug Discov Today Dis Models 2009; 6: 41-45.

7 Tang Q, Bluestone JA: Regulatory T-cell physiology and application to treat autoimmunity. Immunol Rev 2006; 212: 217-237.

8 Eizirik DL, Darville MI: Beta-cell apoptosis and defense mechanisms: lessons from type 1 diabetes. Diabetes 2001; 50 (Suppl 1): S64-S69.

9 von Herrath M: Diabetes: a virus-gene collaboration. Nature 2009; 459: 518-519.

10 Kivity S, Agmon-Levin N, Blank M et al: Infections and autoimmunity - friends or foes? Trends Immunol 2009; 30: 409-414.

11 Bach JF: The effect of infections on susceptibility to autoimmune and allergic diseases. N Engl J Med 2002; 347: 911-920.

12 Zinkernagel RM: Maternal antibodies, childhood infections, and autoimmune diseases. N Engl J Med 2001; 345: 1331-1335.

13 Richardson SJ, Willcox A, Bone AJ et al: The prevalence of enteroviral capsid protein vp1 immunostaining in pancreatic islets in human type 1 diabetes. Diabetologia 2009; 52: $1143-1151$.

14 Oikarinen S, Martiskainen M, Tauriainen S et al: Enterovirus RNA in blood is linked to the development of type 1 diabetes. Diabetes 2011; 60: 276-279.

15 Stene LC, Oikarinen S, Hyöty H et al: Enterovirus infection and progression from islet autoimmunity to type 1 diabetes: the Diabetes and Autoimmunity Study in the Young (DAISY). Diabetes 2010; 59: 3174-3180.

16 Staeva-Vieira T, Peakman M, von Herrath M: Translational mini-review series on type 1 diabetes: Immune-based therapeutic approaches for type 1 diabetes. Clin Exp Immunol 2007; 148: 17-31.

17 Bingley PJ, Williams AJ, Gale EA: Optimized autoantibody-based risk assessment in family members. Implications for future intervention trials. Diabetes Care 1999; 22: 1796-1801.

18 Achenbach P, Bonifacio E, Ziegler AG: Predicting type 1 diabetes. Curr Diab Rep 2005; 5: 98-103. 
19 Cinek O, Kolousková S, Snajderova M et al: HLA class II genetic association of type 1 diabetes mellitus in Czech children. Pediatr Diabetes 2001; 2: 98-102.

20 CODIS (Combined DNA Index System), http://www.fbi.gov/about-us/lab/codis/codis.

21 Vrabelova Z, Kolouskova S, Bohmova K et al: Protein microarray analysis as a tool for monitoring cellular autoreactivity in type 1 diabetes patients and their relatives. Pediatr Diabetes 2007; 8: 252-260.

22 Horvath R, Budinsky V, Kayserova J et al: Kinetics of dendritic cells reconstitution and costimulatory molecules expression after myeloablative allogeneic haematopoetic stem cell transplantation: implications for the development of acute graft-versus host disease. Clin Immunol 2009; 131: 60-69.

23 Parazzini F, Villa A, Moroni S et al: The epidemiology of multiple pregnancies. Acta Genet Med Gemellol (Roma) 1994; 43: 17-23.

24 Eisenbarth GS: Molecular aspects of the etiology of type I diabetes mellitus. J Diabetes Complications 1993; 7: 142-150.

25 von Herrath M: Can we learn from viruses how to prevent type 1 diabetes?: the role of viral infections in the pathogenesis of type 1 diabetes and the development of novel combination therapies. Diabetes 2009; 58: 2-11.

26 Notkins AL: On the track of viruses. Nature 1984; 311: 209-210.

27 Yeung WC, Rawlinson WD, Craig ME: Enterovirus infection and type 1 diabetes mellitus: systematic review and meta-analysis of observational molecular studies. BMJ 2011; 342: d35. doi: 10.1136/bmj.d35.

28 Kawai T, Akira S: TLR signaling. Semin Immunol 2007; 19: 24-32.

29 Kawai T, Akira S: The role of pattern-recognition receptors in innate immunity: update on Toll-like receptors. Nat Immunol 2010; 11: 373-384.

30 Seya T, Oshiumi $\mathrm{H}$, Sasai $\mathrm{M}$ et al: TICAM-1 and TICAM-2: toll-like receptor adapters that participate in induction of type 1 interferons. Int J Biochem Cell Biol 2005; 37: 524-529.

31 Siren J, Pirhonen J, Julkunen I et al: IFN-alpha regulates TLR-dependent gene expression of IFN-alpha, IFN-beta, IL-28, and IL-29. J Immunol 2005; 174: 1932-1937.

32 von Herrath MG, Dockter J, Oldstone MB: How virus induces a rapid or slow onset insulindependent diabetes mellitus in a transgenic model. Immunity 1994; 1: 231-242.

33 Kato H, Takeuchi O, Sato S et al: Differential roles of MDA5 and RIG-I helicases in the recognition of RNA viruses. Nature 2006; 441: 101-105.

34 Meylan E, Tschopp J, Karin M: Intracellular pattern recognition receptors in the host response. Nature 2006; 442: 39-44.

35 Nejentsev S, Walker N, Riches D et al: Rare variants of IFIH1, a gene implicated in antiviral responses, protect against type 1 diabetes. Science 2009; 324: 387-389.
36 Murugaiyan G, Mittal A, Lopez-Diego R et al: IL-27 is a key regulator of IL-10 and IL-17 production by human CD4+ T cells. J Immunol 2009; 183: 2435-2443.

37 Nowak EC, Weaver CT, Turner $\mathrm{H}$ et al: IL-9 as a mediator of Th17-driven inflammatory disease. J Exp Med 2009; 206: 1653-1660.

38 Elyaman W, Bradshaw EM, Uyttenhove C et al: IL-9 induces differentiation of TH17 cells and enhances function of FoxP3+ natural regulatory T cells. Proc Natl Acad Sci USA 2009; 106: 12885-12890.

39 Vrabelova Z, Hrotekova Z, Hladikova Z et al: CD 127- and FoxP3+ expression on CD25+CD4+ T regulatory cells upon specific diabetogeneic stimulation in highrisk relatives of type 1 diabetes mellitus patients. Scand J Immunol 2008; 67: 404-410.

40 Ryden A, Stechova K, Durilova M et al: Switch from a dominant Th1-associated immune profile during the pre-diabetic phase in favour of a temporary increase of a Th3-associated and inflammatory immune profile at the onset of type 1 diabetes. Diabetes Metab Res Rev 2009; 25: 335-343.

41 Stechova K, Bohmova K, Vrabelova Z et al: High T-helper-1 cytokines but low T-helper-3 cytokines, inflammatory cytokines and chemokines in children with high risk of developing type 1 diabetes. Diabetes Metab Res Rev 2007; 23: 462-471.

42 Salminen K, Sadeharju K, Lönnrot M et al: Enterovirus infections are associated with the induction of beta-cell autoimmunity in a prospective birth cohort study. J Med Virol 2003; 69: 91-98.

43 HA Guideline on Enteroviral Infection, 2nd revision www.ha.org.hk/clnguide/ev71v2.htm.

44 Stechova K, Kolar M, Blatny R et al: Healthy first degree relatives of patients with type 1 diabetes exhibit significant differences in basal gene expression pattern of immunocompetent cells compared to controls: expression pattern as predeterminant of autoimmune diabetes. Scand J Immunol 2011; e-pub ahead of print 16 September 2011; doi: 10.1111/j.1365-3083.2011.02637.x.

45 Knip M: Pathogenesis of type 1 diabetes: implications for incidence trends. Horm Res Paediatr 2011; 76 (Suppl 1): 57-64.

46 Redondo MJ, Yu L, Hawa M et al: Heterogeneity of type 1 diabetes. Analysis of monozygotic twins in Great Britain and the United States. Diabetologia 2001; 44: 354-362.

47 Oates NA, van Vliet J, Duffy DL et al: Increased DNA methylation at the AXIN1 gene in a monozygotic twin from a pair discordant for a caudal duplication anomaly. Am J Hum Genet 2006; 79: 155-162.

48 von Herrath MG, Fujinami RS, Whitton JL: Microorganisms and autoimmunity: making the barren field fertile? Nat Rev Microbiol 2003; 1: 151-157.

Supplementary Information accompanies the paper on European Journal of Human Genetics website (http://www.nature.com/ejhg) 